[Supporting Information]

\title{
Synergizing Layered Carbon and Gel Electrolyte for Efficient
}

\section{Energy Storage}

Yanyan Lu, ${ }^{\text {a }}$ Hongyu Mi, ${ }^{*}, \mathrm{a}, \mathrm{b}$ Chenchen Ji, ${ }^{\mathrm{a}}$ Fengjiao Guo, ${ }^{\mathrm{a}}$ Zhengyu Bai, ${ }^{\mathrm{c}}$ Yibo Liu, ${ }^{\mathrm{a}}$

Chang $\mathrm{Yu}^{\mathrm{b}}$ and Jieshan Qiu*,b

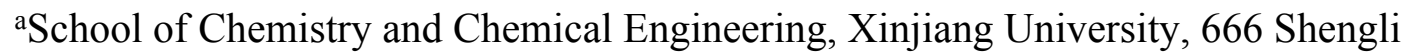

Road, Urumqi 830046, China

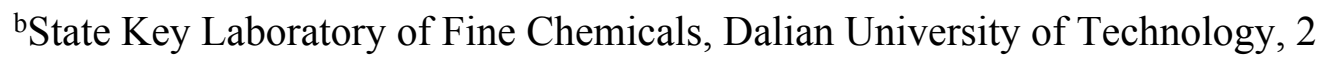

Linggong Road, Dalian 116024, China

'School of Chemistry and Chemical Engineering, Key Laboratory of Green Chemical

Media and Reactions, Ministry of Education, Henan Normal University, 46 East of

Construction Road, Xinxiang 453007, China

${ }^{*}$ Corresponding authors.

E-mail addresses: mmihongyu@163.com (H. Mi), jqiu@dlut.edu.cn (J. Qiu).

Number of pages: 2

Number of Figures: 1 

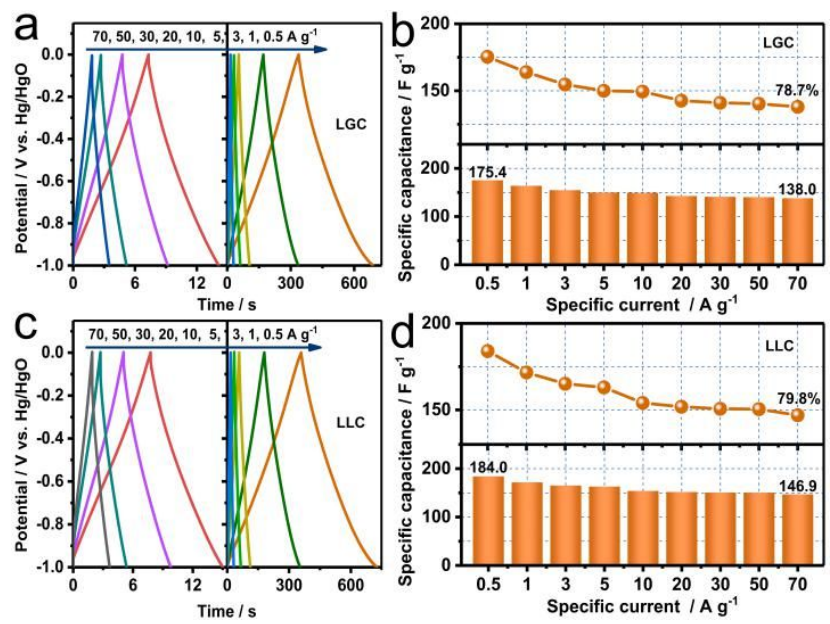

Figure S1. Evaluation of electrode performance on a three-electrode cell in a $6 \mathrm{~mol} \mathrm{~L}^{-1}$ KOH electrolyte: (a, c) GCD curves of LGC and LLC electrodes at specific currents from 0.5 to $70 \mathrm{~A} \mathrm{~g}^{-1}$; (b, d) specific capacitances of LGC and LLC electrodes at specific currents from 0.5 to $70 \mathrm{~A} \mathrm{~g}^{-1}$. 